\title{
Revisión sistemática de estudios de calidad de vida y/o productividad laboral en pacientes tratados con natalizumab
}

\author{
María Echave $^{1} \cdot$ Itziar Oyagüez $^{1} \cdot$ Virginia Casado Ruiz $^{2} \cdot$ Ricardo Ginestal $^{3} \cdot$ Miguel Ángel Casado $^{1}$
}

Published online: 18 January 2018

(c) The Author(s) 2018. This article is published with open access at Springerlink.com

Resumen Introducción La esclerosis múltiple (EM) es una enfermedad incurable, por lo que el objetivo de los tratamientos modificadores de la enfermedad es reducir el número de brotes, enlentecer la progresión de la discapacidad y frenar el deterioro para mejorar la calidad de vida (CV) y la productividad laboral (PL) de los pacientes. El objetivo de este estudio fue realizar una revisión sistemática sobre la evidencia disponible del uso de natalizumab en la PL y/o $\mathrm{CV}$ de los pacientes con EM.

Material y métodos Se realizó una búsqueda sistemática en bases de datos nacionales (IBECS, IME), e internacionales (MEDLINE, mediante PUBMED) y la Biblioteca Cochrane. Se incluyeron aquellos estudios que evaluaran la PL y/o CV, medida esta a través de instrumentos validados en pacientes con EM tratados con natalizumab.

Resultados La búsqueda inicial arrojó 84 artículos, de los que se incluyeron 18. Cuatro eran modelizaciones, seis estudios evaluaban la CV en práctica clínica habitual, tres evaluaban resultados reportados por pacientes y uno evaluaba la PL. Dos artículos adicionales eran análisis conjuntos de los estudios AFFIRM y SENTINEL, y los tres restantes eran análisis post-hoc del estudio AFFIRM, ambos estudios de fase III de natalizumab. En todos ellos la CV y la PL de los pacientes tratados con natalizumab mejoraron significativamente, medida la primera bien como aumento de años de vida ajustados por calidad bien por mejora de las puntuaciones basales analizadas con los cuestionarios validados habi-

M. Echave

mechave@porib.com

1 Pharmacoeconomics \& Outcomes Research Iberia (PORIB), Madrid, España

2 Servicio de Neurología, Hospital de Mataró, Mataró, Barcelona, España

3 Servicio de Neurología, Fundación Jiménez Díaz, Madrid, España tuales, tanto generales como específicos de EM, y la segunda como aumento de horas trabajadas.

Conclusión Los resultados de los estudios revisados, a pesar de la diversidad de su metodología, sugieren que natalizumab mejora la CV y PL de los pacientes con EM.

Palabras clave Natalizumab - Calidad de vida - Esclerosis múltiple $\cdot$ Productividad laboral

Abstract Introduction Multiple sclerosis (MS) is an incurable disease and the main goal of disease-modifying treatments (DMT) is to reduce the number of relapses and slow the progression of physical and cognitive disabilities. Consequently, DMT would prevent worsening of disability and improve patients' quality of life (QoL) and work productivity. The aim of this study was to conduct a systematic review of the clinical evidence regarding effect of natalizumab o QoL and work productivity on patients with MS.

Material and methods A systematic literature search was conducted in National Spanish database ICEBS and IME, and international in MEDLINE through PubMed seeker and the Cochrane Library. Studies assessing work productivity and/or QoL, measured by validated instruments, in MS patients treated with natalizumab were included.

Results The initial search yielded 84 articles, of which 18 were included. Four were Markov model, six studies evaluated QoL in routine clinical practice, three assessed results reported by patients and one evaluated work productivity. Two remaining were metaanalysis of the AFFIRM and SENTINEL studies, and three additional ones were provided by post-hoc analysis of the AFFIRM trial, phase III studies of natalizumab. In all studies retrieved, the QoL (measured as increase in quality-adjusted life years [QALYs] or as improvement from baseline scores analyzed with validated questionnaires) and work productivity of patients (measured as increased worked hours) treated with 
natalizumab improved significantly, both general and MS specific.

Conclusion Despite the diversity of methodologies among the included studies, treatment with natalizumab was associated with improvement in the QoL and work productivity in patients with MS.

Keywords Natalizumab · Quality of life · Multiple sclerosis · Work productivity

\section{Introducción}

La esclerosis múltiple (EM) es una enfermedad crónica de base inmunitaria, inflamatoria y degenerativa del sistema nervioso central causada por desmielinización inflamatoria y daño axonal [1] que se caracteriza por una discapacidad acumulativa. Al diagnóstico un $85 \%$ de los pacientes presenta un subtipo, denominado EM remitente recurrente (EMRR), donde la desmielinización coincide con la aparición de brotes o recidivas periódicas [2]. La medida de discapacidad se puede establecer a través de distintos métodos, uno de ellos mediante la Expanded Disability Status Scale (EDSS), una escala de 10 puntos que mide diferentes áreas de discapacidad funcional [3]. A diferencia de otras enfermedades neurodegenerativas, la EM afecta generalmente a adultos jóvenes, laboralmente activos. Concretamente, a nivel mundial, la edad promedio de inicio se sitúa en los 29,2 años [4] y es la principal causa de morbilidad no traumática del sistema nervioso central en adultos jóvenes [5]. Este hecho explica la importante repercusión de la EM sobre la productividad laboral de los pacientes.

Tanto las recurrencias episódicas, denominadas brotes, como las secuelas crónicas de esta enfermedad interfieren en las actividades diarias del paciente, disminuyendo así su calidad de vida relacionada con la salud (CVRS). Este impacto se acentúa con el avance o progresión de la enfermedad debido a un aumento de la discapacidad física y deterioro cognitivo, entre otros factores [6, 7]. En general, los estudios indican una disminución del $30 \%$ de la función física en EM leve, aumentando hasta el $40 \%$ para EM moderada y $50 \%$ en los casos graves [8].

En el ámbito de enfermedades crónicas como la EM, para la cual no existe curación y donde el objetivo del tratamiento es atenuar síntomas, evitar complicaciones y mejorar el bienestar de los pacientes, las medidas clásicas de resultados en salud (mortalidad, morbilidad y expectativa de vida) no son suficientes, de ahí que resulte necesario la incorporación de la CVRS como medida necesaria en la evaluación clínica de un medicamento, que ha supuesto una gran innovación en este campo [9].

La CVRS engloba la valoración de los dominios físico, emocional y social de la salud [10]. Los instrumentos de medida suelen ser cuestionarios validados que, con frecuencia, contienen varias escalas que se corresponden con los distintos dominios. Por ello, según el tipo de dominio evaluado puede llevar al desarrollo de cuestionarios, bien genéricos o específicos de una enfermedad en concreto. Determinados cuestionarios genéricos como el EuroQol-5D o Short Form 36 (SF-36), o específicos de EM, como el Multiple Sclerosis Impact Scale 29 (MSIS-29) o la Multiple Sclerosis Quality of Life Inventory (MSQLI) permiten obtener un valor agregado de la CVRS en forma de utilidad, que posteriormente permite transformar los años de vida ganados en años de vida ajustados por calidad (AVAC), como medida adicional de eficacia, equivalentes a un año de vida en perfecto estado de salud.

Una reciente revisión sistemática realizada en EM por la Agencia Canadiense de Evaluación de Medicamentos y Tecnologías Sanitarias desarrollada en el año 2013 [2], concluyó que, de todos los medicamentos aprobados en el tratamiento de la EM, solo había datos disponibles de CVRS de interferón beta 1a intramuscular (IFN $\beta$-1a IM) y de natalizumab.

Natalizumab (Tysabri ${ }^{\circledR}$, Biogen) está indicado en el tratamiento de la EMRR en pacientes que no hayan respondido al tratamiento con una primera línea o en pacientes que presentan formas inicialmente agresivas de la enfermedad [11]. Los resultados de los ensayos clínicos en fase III [12, 13] demostraron que la terapia con natalizumab reduce significativamente la tasa anual de brotes y la discapacidad. Asimismo, tiene un perfil beneficio-riesgo favorable [14], por lo que es un tratamiento de elección en pacientes con EM en los que fracasan otros tratamientos o que comienzan con una enfermedad grave de evolución rápida.

El objetivo de este estudio fue revisar de forma sistemática la evidencia publicada disponible sobre el impacto en la calidad de vida y en productividad laboral en pacientes con EM tratados con natalizumab.

\section{Métodos}

\section{Búsqueda}

Se realizó una búsqueda sistemática en las bases de datos nacionales IBECS (Índice bibliográfico español en ciencias de la salud) e IME (Índice médico español), e internacionales MEDLINE a través del buscador PUBMED y en la Biblioteca Cochrane, que engloba la base de datos Cochrane de revisiones sistemáticas, la base de datos de evaluación de tecnologías sanitarias y la base de datos de evaluación económica del NHS, utilizando las palabras clave (medical subject headings [MeSH]): "natalizumab" AND-“multiple sclerosis" AND-("quality of life" OR "productivity"-OR "work productivity"). La estrategia de búsqueda se recoge en la Tabla 1.

La búsqueda final se realizó el día 6 de marzo de 2017. Se orientó la búsqueda a estudios publicados que presentaran 
Tabla 1 Estrategia de búsqueda realizada en PUBMED a través de MEDLINE

\begin{tabular}{|c|c|c|c|}
\hline & Término & Detalles de la búsqueda (6 mar 2017) & $\begin{array}{l}\text { N. } \\
\text { artículos }^{\circ}\end{array}$ \\
\hline & Producto & $\begin{array}{l}\text { "natalizumab" [Supplementary Concept] OR "natalizumab" [All Fields] OR } \\
\text { "tysabri” [All Fields] }\end{array}$ & 1.939 \\
\hline & Enfermedad & multiple sclerosis [All fields] & 72.847 \\
\hline A & $\begin{array}{l}\text { Enfermedad y } \\
\text { producto }\end{array}$ & $\begin{array}{l}\text { ((“"natalizumab" [Supplementary Concept] OR "natalizumab” [All Fields] OR } \\
\text { "tysabri” [All Fields])) AND multiple sclerosis [All fields]) }\end{array}$ & 1.531 \\
\hline 1 & Quality of life & $\begin{array}{l}\text { "quality of life" [MeSH Terms] OR ("quality" [All Fields] AND "life" [All Fields]) } \\
\text { OR "quality of life" [All Fields] }\end{array}$ & 292.610 \\
\hline 2 & $\begin{array}{l}\text { Health-related } \\
\text { quality of life }\end{array}$ & $\begin{array}{l}\text { health-related [All Fields] AND quality [All Fields] AND ("life" [MeSH Terms] } \\
\text { OR "life" [All Fields]) }\end{array}$ & 33.750 \\
\hline B & 1 or 2 & $\begin{array}{l}\text { ((health-related [All Fields] AND quality [All Fields] AND ("life" [MeSH Terms] } \\
\text { OR "life" [All Fields]))) OR ("quality of life" [MeSH Terms] OR ("quality" [All } \\
\text { Fields] AND "life" [All Fields]) OR "quality of life" [All Fields]) }\end{array}$ & 292.610 \\
\hline 3 & $\begin{array}{l}\text { Work } \\
\text { productivity } \\
\text { loss }\end{array}$ & $\begin{array}{l}\text { (“work” [MeSH Terms] OR “work" [All Fields]) AND "productivity" [All Fields] } \\
\text { AND loss [All Fields] }\end{array}$ & 961 \\
\hline 4 & Productivity & “efficiency” [MeSH Terms] OR “efficiency" [All Fields] & 349.038 \\
\hline $\mathrm{C}$ & 3 or 4 & $\begin{array}{l}((((\text { "work" [MeSH Terms] OR “work" [All Fields]) AND "productivity" [All } \\
\text { Fields] AND loss [All Fields]))) OR ("efficiency" [MeSH Terms] OR "efficiency" } \\
\text { [All Fields]) }\end{array}$ & 349.688 \\
\hline $\mathrm{D}$ & $\mathrm{B}$ or $\mathrm{C}$ & $\begin{array}{l}((((\text { health-related [All Fields] AND quality [All Fields] AND ("life" [MeSH Terms] } \\
\text { OR "life" [All Fields]))) OR ("quality of life" [MeSH Terms] OR ("quality" [All } \\
\text { Fields] AND "life" [All Fields]) OR "quality of life" [All Fields]))) OR ((((("work" } \\
\text { [MeSH Terms] OR "work" [All Fields]) AND "productivity" [All Fields] AND loss } \\
\text { [All Fields]))) OR ("efficiency" [MeSH Terms] OR "efficiency" [All Fields])) }\end{array}$ & 637.808 \\
\hline $\mathrm{E}$ & $\mathrm{D}$ and $\mathrm{A}$ & $\begin{array}{l}((((((\text { health-related [All Fields] AND quality [All Fields] AND ("life" [MeSH } \\
\text { Terms] OR "life" [All Fields]))) OR ("quality of life" [MeSH Terms] OR (“quality" } \\
\text { [All Fields] AND "life" [All Fields]) OR "quality of life" [All Fields]))) OR } \\
(((((\text { "work" [MeSH Terms] OR "work" [All Fields]) AND "productivity" [All } \\
\text { Fields] AND loss [All Fields]))) OR ("efficiency" [MeSH Terms] OR "efficiency" } \\
\text { [All Fields])))) AND (((("natalizumab" [Supplementary Concept] OR } \\
\text { "natalizumab" [All Fields] OR "tysabri" [All Fields])) AND multiple sclerosis [All } \\
\text { fields])) }\end{array}$ & 85 \\
\hline $\mathrm{F}$ & $\begin{array}{l}\text { E and ((english } \\
\text { [lang] OR } \\
\text { spanish [lang])) }\end{array}$ & 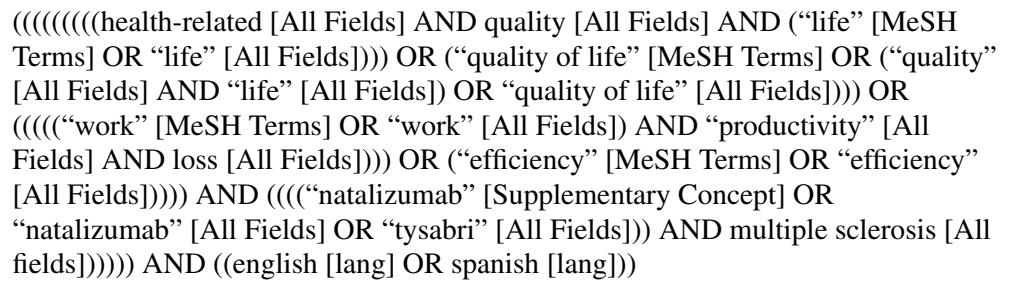 & 80 \\
\hline G & & F AND Filters: Publication date to $2016 / 12 / 31$ & 76 \\
\hline
\end{tabular}

evaluación en términos de calidad de vida y/o productividad laboral en pacientes con EM que recibieran tratamiento con natalizumab. Se llevó a cabo un segundo filtro de las citas que aparecían referenciadas en los artículos revisados en la búsqueda inicial, con el fin de asegurar que los estudios más relevantes fueran incluidos.

\section{Selección}

Se estableció como límite temporal de publicación el día 31 de diciembre de 2016, por lo que se consideraron incluidos todos aquellos artículos publicados hasta esa fecha.
Como otros criterios de inclusión se consideraron artículos escritos tanto en lengua inglesa como española publicados hasta ese momento, investigaciones que midieran el cambio en la CVRS del paciente desde el momento basal hasta haber recibido tratamiento con natalizumab a través de instrumentos validados. Así mismo se consideró como criterio de inclusión publicaciones que aportaran información sobre la productividad laboral (absentismo, jubilación prematura, etc.) en pacientes con EM.

Como criterios de exclusión se descartaron artículos donde natalizumab fuera administrado para el tratamiento 


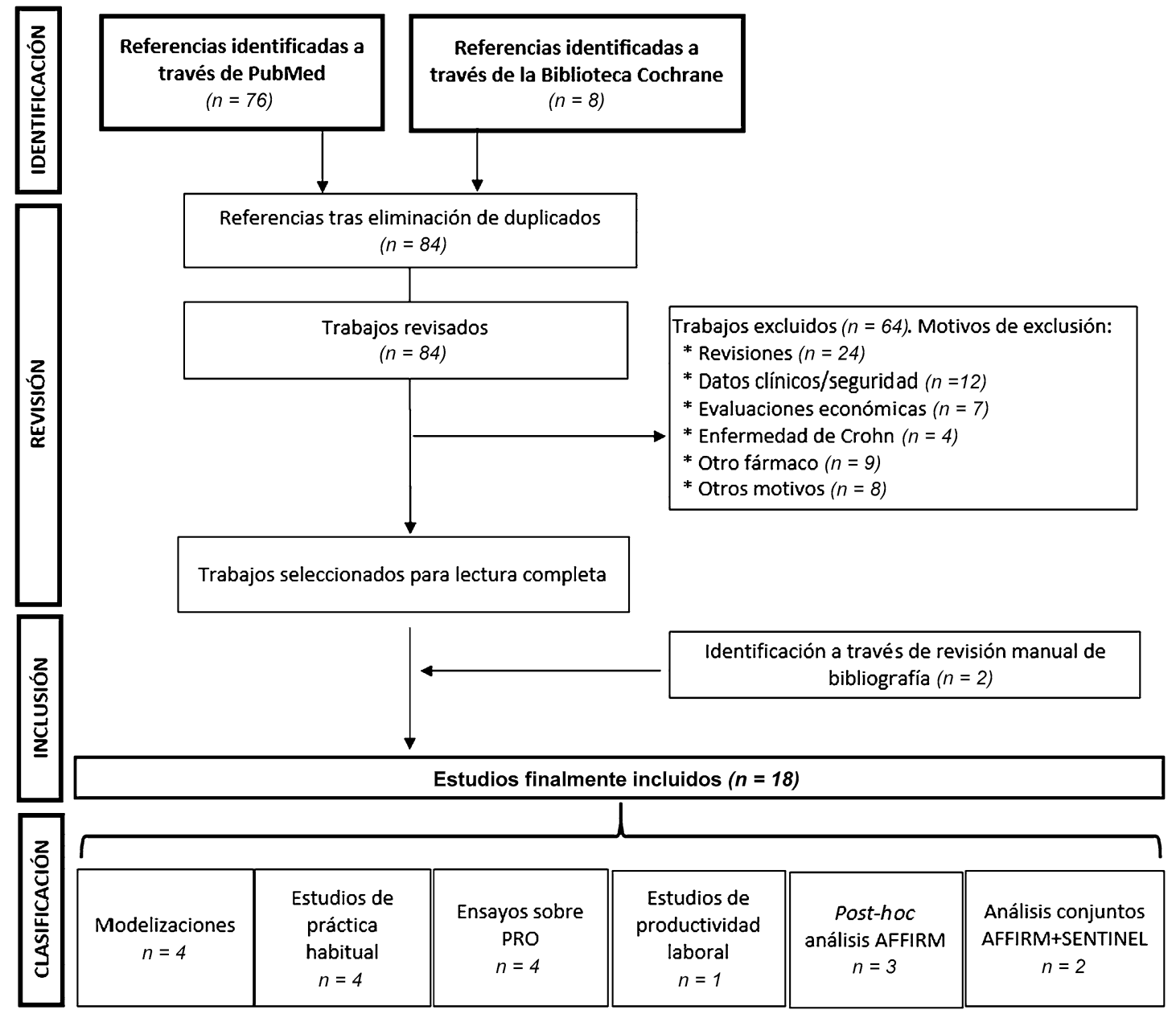

Figura 1 Representación esquemática del proceso de búsqueda bibliográfica. PRO: patient reported outcomes

de otras enfermedades que no fueran EM, tanto dentro de indicación (enfermedad de Crohn en Estados Unidos) como en el tratamiento de otras patologías.

\section{Extracción de datos}

Dos revisores examinaron las referencias basadas en los criterios de inclusión definidos y extrajeron los datos. Los datos fueron extraídos por un autor y comprobados por un segundo autor. Las posibles discrepancias fueron resueltas mediante revisión conjunta y discusión entre ambos revisores.

\section{Resultados}

En la Figura 1 se recoge gráficamente el proceso de selección de los estudios. En la búsqueda inicial se identificaron 84 trabajos potenciales hasta el día 31 de diciembre de 2016 (76 a través de MEDLINE y 8 de la Biblioteca Cochrane). Del total de artículos se descartaron hasta 68 por diversos motivos, en concreto cuatro se identificaron como duplicados. La mayoría de los trabajos, el 35,3\% $(n=24)$, fueron excluidos por tratarse de revisiones sistemáticas, seguidos por estudios ( $n=12$ ) que evaluaban únicamente la eficacia y seguridad de natalizumab (17,6\%), o artículos que correspondían solo a evaluaciones económicas, el 10,3\% $(n=7)$. Otros motivos de exclusión fueron la obtención de criterios de CVRS sin la utilización de instrumentos validados o cuyos autores se limitaron a realizar un análisis descriptivo de las respuestas por dimensiones, u otro tipo de análisis sin llegar a calcular puntuaciones concretas, o sin establecer comparaciones entre cortes temporales del tratamiento con natalizumab $(n=8 ; 11,7 \%)$. Pese a filtrar la búsqueda por patología concreta, cuatro artículos $(5,9 \%)$ correspondían a la evaluación de natalizumab en enfermedad de Crohn, considerado como criterio de exclusión, y 9 (13,2\%) se referían a otro producto diferente a natalizumab.

Tras el proceso de selección inicial, durante la revisión manual de citas bibliográficas se detectaron dos artículos adicionales. 


\section{Estudios incluidos}

Finalmente se incluyeron un total de 18 estudios, de los cuales cuatro $(22,2 \%)$ eran modelizaciones [15-18], seis estudios evaluaban la calidad de vida en condiciones de práctica clínica habitual tras la administración de natalizumab [19-24], tres eran ensayos que evaluaban diferentes medidas de salud reportadas por pacientes (patient reported outcomes [PRO]) [25-27] y un estudio evaluaba el efecto de natalizumab sobre la productividad laboral en Suecia [28]. De los restantes dos eran análisis conjuntos $[29,30]$ de los estudios AFFIRM y SENTINEL, estudios clínicos de natalizumab, focalizándose concretamente en evaluar la CVRS, y en tres se aportaban post-hoc análisis del ensayo AFFIRM que relacionaban la capacidad visual [31] y la velocidad de desplazamiento $[23,32]$ con la calidad de vida.

\section{Modelizaciones}

Las cuatro modelizaciones incluidas en esta revisión emplearon un modelo de Markov para evaluar los AVAC ganados tras el tratamiento con natalizumab. Los modelos de Markov son un tipo de modelo de decisión que se usa para simular transiciones entre diferentes estados de salud a lo largo del tiempo [33]. Todos los modelos coincidieron en la definición de estados de salud en la EM según la escala EDSS.

Una de las simulaciones se realizó sobre datos de población de Inglaterra y Gales [17] y las otras tres sobre población de Estados Unidos [15, 16, 18]. Las utilidades empleadas en dichos estudios se obtuvieron de investigaciones realizadas en los propios países, Reino Unido y Estados Unidos respectivamente, aspecto destacable, ya que se ha confirmado que el valor de utilidad para un mismo estado de salud puede variar sustancialmente entre distintos países y culturas, aunque se hayan empleado los mismos métodos y cuestionarios para su obtención [34]. Las características de estos cuatro estudios se recogen en la Tabla 2.

El objetivo de los estudios [15-18] fue evaluar el balance beneficio-riesgo del tratamiento con natalizumab en pacientes con EM, principalmente orientado al riesgo de sufrir leucoencefalopatía multifocal progresiva (LMP), un acontecimiento adverso (AA) que se ha asociado al tratamiento prolongado con natalizumab [12]. Los horizontes temporales para el análisis variaron entre dos $[15,17]$ y 20 años [16-18].

Para estimar la ganancia de AVAC adicionales que supone la administración de natalizumab, las alternativas terapéuticas frente a las que se comparó fueron IFN $\beta$ [17] e IFN $\beta$-1a [16], acetato de glatirámero (AG) [18] y fingolimod [17, 18]. Adicionalmente, el modelo inglés incluyó la opción de no recibir tratamiento [17] mientras que Thompson y cols. [16] incluyeron la opción de no recibir tratamiento y la alternativa denominada como "perfecto tratamiento posible para la EM", que consistía en mantener el estado basal de los pacientes respecto al nivel de discapacidad y la utilidad durante todo el horizonte temporal que durara el análisis. La eficacia de natalizumab en los cuatro casos se extrajo del estudio AFFIRM (riesgo anual para desarrollar LMP: 0,067\%) [12] y el riesgo de los pacientes de sufrir LMP se obtuvo, en dos trabajos [17, 18], de la publicación más reciente y actual en ese momento del riesgo de padecer LMP (el riesgo estimado estaba comprendido entre $\leq 0,09 / 1.000$ en pacientes anti-JCV negativos, y $11,1 / 1.000$ en pacientes en tratamiento con natalizumab durante más de 2 años, que habían tomado previamente inmunosupresores y además eran anti-JCV positivos) [35]. En el caso de los otros dos estudios estadounidenses $[15,16]$ se basaron en una misma publicación que estimaba el riesgo de sufrir LMP en el año 2006 [36].

El estudio inglés [17] dividía a los pacientes en 3 grupos según la presencia o ausencia de anticuerpos anti-JCV. Un grupo tenía anticuerpos negativos, y los otros dos positivos con y sin historia previa de tratamiento con inmunosupresores, siendo la presencia de dichos anticuerpos un factor de riesgo asociado para desarrollar LMP. Los resultados fueron que natalizumab aportaba más AVAC frente a todos los comparadores analizados (fingolimod, IFN $\beta$ e IFN $\beta$-1a y no recibir tratamiento) y en todos los subgrupos de riesgo de sufrir LMP, tanto a dos como a 20 años. En el estudio de Thompson y cols. [16] se obtuvo que, en un horizonte temporal de 20 años, natalizumab aportaría un total de 9,50 AVAC, que suponen 0,80 AVAC adicionales frente a la opción de no recibir tratamiento, es decir, frente al curso natural de la enfermedad (8,70 AVAC) y 0,38 AVAC adicionales frente a IFN $\beta-1 \mathrm{a}(9,12$ AVAC). La opción de "posible tratamiento perfecto para la EM" aportaba 10,59 AVAC, 1,09 AVAC adicionales frente a natalizumab [16]. La pérdida en salud producida por LMP fue baja ( $-0,06$ AVAC), y aunque se aumentó el riesgo de sufrir LMP en el modelo, se concluyó que habría que aumentar casi siete veces este riesgo para que natalizumab produjera menos beneficios en salud que IFN $\beta-1 \mathrm{a}$ [16].

Campbell y cols. [18] determinaron que a lo largo de 20 años la terapia de natalizumab se asociaría con 1,2 y 0,4 AVAC incrementales frente a AG y fingolimod, respectivamente. Además, con natalizumab se obtendrían más tiempo libre de discapacidad $(0,61$ años frente a $A G$ y 0,11 años frente a fingolimod), aunque se observó también un aumento del riesgo de LMP $(0,0165$ por paciente tratado con natalizumab) que fue considerado marginal. La probabilidad de que natalizumab resultara una alternativa más efectiva en términos de AVAC incrementales se estimó en 0,963 frente a AG y 0,720 frente a fingolimod.

El restante estudio [15] modelizó, a partir de los estudios pivotales, la ganancia en AVAC derivada de la administración tanto de natalizumab como de IFN $\beta$-1a en un horizonte 
Tabla 2 Características de los modelos que evaluaban los años de vida ajustados por calidad aportados con natalizumab

\begin{tabular}{|c|c|c|c|c|}
\hline & Walker 2014 [17] & Thompson 2008 [16] & Dorsey 2007 [15] & Campbell 2013 [18] \\
\hline $\begin{array}{l}\text { Objetivo del } \\
\text { estudio }\end{array}$ & $\begin{array}{l}\text { Modelizar los riesgos y } \\
\text { beneficios en salud del } \\
\text { tratamiento con natalizumab en } \\
\text { función del riesgo de sufrir } \\
\text { LMP a corto y largo plazo }\end{array}$ & $\begin{array}{l}\text { Modelizar riesgos y } \\
\text { beneficios en salud del } \\
\text { tratamiento con } \\
\text { natalizumab a largo plazo }\end{array}$ & $\begin{array}{l}\text { Modelizar riesgos y } \\
\text { beneficios en salud del } \\
\text { tratamiento con } \\
\text { natalizumab a corto plazo }\end{array}$ & $\begin{array}{l}\text { Modelizar riesgos y } \\
\text { beneficios en salud del } \\
\text { tratamiento con } \\
\text { natalizumab a largo plazo }\end{array}$ \\
\hline Comparadores & $\begin{array}{l}\text { Fingolimod, IFN } \beta \text { (todos) y sin } \\
\text { tratamiento }\end{array}$ & $\begin{array}{l}\text { Sin tratamiento, IFN } \beta-1 \mathrm{a} \\
\text { y un hipotético "perfecto } \\
\text { tratamiento posible para } \\
\text { EM" }\end{array}$ & IFN $\beta-1 \mathrm{a}$ & $\begin{array}{l}\text { Acetato de glatirámero y } \\
\text { fingolimod }\end{array}$ \\
\hline $\begin{array}{l}\text { Horizonte } \\
\text { temporal y tasa } \\
\text { de descuento para } \\
\text { los resultados en } \\
\text { salud }\end{array}$ & Dos y 20 años; $3,5 \%$. & 20 años; $3,0 \%$. & $\begin{array}{l}\text { Dos años, sin tasa de } \\
\text { descuento por el corto } \\
\text { horizonte temporal }\end{array}$ & 20 años; $0 \%$ \\
\hline $\begin{array}{l}\text { Características de } \\
\text { los pacientes con } \\
\text { EM analizados }\end{array}$ & $\begin{array}{l}\text { Pacientes naïve, } 34,5 \text { años, } \\
77,8 \% \text { de mujeres, } 5 \text { años desde } \\
\text { diagnóstico, distribución por } \\
\text { EDSS según estudio } \\
\text { AFFIRM [12] }\end{array}$ & $\begin{array}{l}30 \text { años, } 66 \% \text { de mujeres, } \\
\text { distribución por EDSS } \\
\text { según datos de la historia } \\
\text { natural de EM }\end{array}$ & $\begin{array}{l}\text { Datos de eficacia } \\
\text { (reducción absoluta del } \\
\text { riesgo) derivada de los } \\
\text { pacientes de los estudios } \\
\text { AFFIRM y PRISMS para } \\
\text { natalizumab e IFN } \beta-1 \mathrm{a}, \\
\text { respectivamente }\end{array}$ & $\begin{array}{l}\text { Pacientes naïve, } 30 \text { años, } \\
\text { distribución por EDSS } \\
\text { según datos de historia } \\
\text { natural de EM (cohorte } \\
\text { London, Ontario) }\end{array}$ \\
\hline $\begin{array}{l}\text { Diseño del } \\
\text { estudio }\end{array}$ & $\begin{array}{l}\text { Modelo de Markov con } 21 \\
\text { estados de salud, } 10 \text { para EMRR } \\
\text { y } 10 \text { para EMSP y uno para } \\
\text { muerte } \\
\text { Ciclos de un año de duración }\end{array}$ & $\begin{array}{l}\text { Modelo de Markov } \\
\text { Ciclos de seis meses de } \\
\text { duración }\end{array}$ & $\begin{array}{l}\text { Modelo de Markov que } \\
\text { evaluaba los AVAC } \\
\text { aportados tanto por } \\
\text { natalizumab como por } \\
\text { IFN } \beta \text {-1a }\end{array}$ & $\begin{array}{l}\text { Modelo de Markov. } \\
\text { Ciclos de un año de } \\
\text { duración }\end{array}$ \\
\hline Utilidades & $\begin{array}{l}\text { Extraídas de encuesta de EM en } \\
\text { Reino Unido }\end{array}$ & $\begin{array}{l}\text { Extraídas de un estudio } \\
\text { de preferencia de } \\
\text { pacientes en Norte } \\
\text { América }\end{array}$ & $\begin{array}{l}\text { Extraídas de un estudio } \\
\text { de preferencia de } \\
\text { pacientes en Norte } \\
\text { América y un análisis } \\
\text { coste-efectividad }\end{array}$ & Obtenidas de literatura \\
\hline $\begin{array}{l}\text { Medida de } \\
\text { resultados }\end{array}$ & $\begin{array}{l}\text { AVAC totales dividiendo los } \\
\text { pacientes por subgrupos de } \\
\text { riesgo de sufrir LMP }\end{array}$ & AVAC & AVAC & $\begin{array}{l}\text { AVAC, tiempo sin } \\
\text { discapacidad }\end{array}$ \\
\hline $\begin{array}{l}\text { Análisis de } \\
\text { sensibilidad }\end{array}$ & Sí & Sí & No & Sí \\
\hline \multirow[t]{2}{*}{ Resultados } & $\begin{array}{l}\text { Horizonte temporal } 2 \text { años: } \\
\text { natalizumab aporta } 1,303 ; 1,302 \\
\text { y } 1,300 \text { AVAC/paciente (según } \\
\text { grupos de menor a mayor riesgo } \\
\text { de sufrir LMP) frente a } 1,287 \\
\text { aportados por fingolimod, } 1,217 \\
\text { por IFN } \beta \text { y } 1,283 \text { con la opción } \\
\text { de no tratamiento, } \\
\text { respectivamente }\end{array}$ & \multirow[t]{2}{*}{$\begin{array}{l}\text { Natalizumab aporta } 0,80 \\
\text { AVAC extra/paciente }(9,50 \\
\text { AVAC) frente al curso } \\
\text { natural de la enfermedad } \\
(8,70 \text { AVAC). Frente al } \\
\text { "hipotético tratamiento } \\
\text { perfecto para EM", } \\
\text { natalizumab resultaría en un } \\
43 \% \text { del mejor tratamiento } \\
\text { posible frente a un } 22 \% \text { del } \\
\text { IFN } \beta-1 \text { a }\end{array}$} & \multirow[t]{2}{*}{$\begin{array}{l}\text { A dos años natalizumab } \\
\text { aporta } 0,033 \text { AVAC/paciente } \\
\text { con una mínima pérdida de } \\
0,001 \text { AVAC debido a LMP. } \\
\text { El tratamiento con IFN } \beta-1 \mathrm{a} \\
\text { aporta } 0,033 \text { AVAC }\end{array}$} & $\begin{array}{l}\text { Horizonte temporal } 20 \\
\text { años: natalizumab a lo } \\
\text { largo de } 20 \text { años aporta } \\
1,2 \text { y } 0,4 \text { AVAC } \\
\text { extra/paciente y } 0,61 \text { y } \\
0,11 \text { años libres de } \\
\text { discapacidad } \\
\text { adicionales/paciente } \\
\text { frente a AG y fingolimod, } \\
\text { respectivamente. La } \\
\text { probabilidad de AVAC } \\
\text { incrementales de } \\
\text { natalizumab fue } 0,963 \\
\text { frente a AG y } 0,720 \\
\text { frente a fingolimod }\end{array}$ \\
\hline & $\begin{array}{l}\text { Horizonte temporal } 20 \text { años: } \\
\text { natalizumab aporta } 6,538 ; 6,396 \\
\text { y } 6,194 \text { AVAC (según grupos de } \\
\text { menor a mayor riesgo de sufrir } \\
\text { LMP) frente a } 6,072 \text { aportados } \\
\text { por fingolimod, } 6,018 \text { por IFN } \beta \\
\text { y } 5,820 \text { con la opción de no } \\
\text { tratamiento, respectivamente }\end{array}$ & & & $\begin{array}{l}\text { Horizonte temporal dos } \\
\text { años: natalizumab aporta } \\
0,04 \text { y } 0,01 \text { AVAC } \\
\text { extra/paciente frente a } \\
\text { AG y fingolimod } \\
\text { respectivamente }\end{array}$ \\
\hline
\end{tabular}


Tabla 2 (Continuación)

\begin{tabular}{|c|c|c|c|c|}
\hline & Walker 2014 [17] & Thompson 2008 [16] & Dorsey 2007 [15] & Campbell 2013 [18] \\
\hline Conclusiones & $\begin{array}{l}\text { Natalizumab aporta mayor } \\
\text { número de AVAC comparado } \\
\text { con fingolimod, IFN } \beta \text { y no } \\
\text { recibir tratamiento, aun cuando } \\
\text { el riesgo de sufrir LMP se tiene } \\
\text { en cuenta }\end{array}$ & $\begin{array}{l}\text { Se necesitaría } \\
\text { incrementar más de siete } \\
\text { veces el riesgo actual de } \\
\text { sufrir LMP con } \\
\text { natalizumab para } \\
\text { disminuir los AVAC } \\
\text { aportados por debajo de } \\
\text { IFN } \beta-1 \text { a }\end{array}$ & $\begin{array}{l}\text { Durante los dos primeros } \\
\text { años de tratamiento con } \\
\text { natalizumab el beneficio } \\
\text { aportado por el } \\
\text { tratamiento es mucho } \\
\text { mayor que el riesgo de } \\
\text { LMP }\end{array}$ & $\begin{array}{l}\text { Natalizumab aporta } \\
\text { mayores beneficios a } \\
\text { largo plazo, con un } \\
\text { incremento mínimo del } \\
\text { riesgo de LMP en } \\
\text { pacientes anti-JCV } \\
\text { negativos }\end{array}$ \\
\hline
\end{tabular}

AVAC: años de vida ajustados por calidad; EM: esclerosis múltiple; IFN $\beta$ : interferón beta; LMP: leucoencefalopatía multifocal progresiva

temporal de dos años, obteniendo como resultado en ambos casos 0,033 AVAC adicionales con una mínima pérdida de 0,001 AVAC debido a LMP [15].

En los análisis de sensibilidad del estudio de Thompson y cols. [16] se aumentó la disutilidad asociada a LMP y se demostró que los resultados para natalizumab en términos de AVAC no eran sensibles a esta variación, ya que supuso una disminución de 0,01 y 0,03 en el grupo de pacientes con anticuerpos anti-JCV positivos, sin y con tratamiento previo con inmunosupresores respectivamente [17].

De los resultados de las modelizaciones se desprende que la ganancia en AVAC en los pacientes que reciben tratamiento con natalizumab, en horizontes temporales comprendidos entre 2 y 20 años, es mayor que con los comparadores analizados. En relación con el riesgo de sufrir LMP habría que aumentar de forma bastante considerable el mismo para que la ganancia en AVAC fuera menor y el beneficio del tratamiento no se viera compensado por el riesgo que supone padecer este AA.

\section{Estudios de calidad de vida relacionada con la salud en la práctica clínica habitual y ensayos de medidas de salud percibidas por los pacientes}

De los estudios con datos sobre CVRS en la práctica habitual el estudio TYNERGY [21] y la investigación de Putzki y cols. [20], cuyo objetivo principal en ambos casos era evaluar la fatiga relacionada con la EM, seis meses después de haber iniciado el tratamiento con natalizumab, incluyeron como objetivo secundario la medida de la CVRS utilizando el cuestionario SF-12 y una escala visual analógica (EVA), respectivamente. Asimismo se analizaron los $\mathrm{PRO}$ incluidos en el programa TOUCH al inicio y después de tres, seis y 12 meses del tratamiento con natalizumab, que incluyeron CVRS general y específica de la EM, capacidad de realizar actividades cotidianas, nivel de discapacidad, función cognitiva y fatiga [19, 22]. Las características de los tres estudios se recogen en la Tabla 3 .

En el estudio TOUCH [22] se comunicaron mejoras estadísticamente significativas tanto en el componente físico (CSF) $(p<0,01)$ como en el componente mental (CSM) $(p<0,001)$ al responder al cuestionario general de CVRS (SF-12), así como el cuestionario específico de la EM (MSIS-29). Las mejoras se observaron a partir de la primera evaluación, a los tres meses de haber comenzado el tratamiento con natalizumab. Así mismo el impacto de la EM en la funcionalidad cognitiva y en la fatiga se redujeron significativamente $(p<0,001$ en ambas) [22]. La mejoría en los resultados percibidos por el paciente se observó a los tres meses, y se mantuvo hasta los 12 meses que duraba el estudio. Tanto la fatiga relacionada con la EM, como el resto de variables evaluadas mejoraron de forma significativa respecto al estado basal, tanto en el estudio TYNERGY [21] como en la investigación realizada sobre 42 pacientes durante 6 meses [20] ( $p<0,0001$ y $p<0,01$, respectivamente).

La evaluación de 6 meses de tratamiento con natalizumab sobre parámetros relacionados con el sueño, realizada en el estudio NAPS-MS [26], evidenció mejoras significativas respecto al valor basal en los niveles de fatiga, determinados con la escala de severidad de la fatiga (Fatigue Severity Scale) $(p=0,03)$ y una EVA de fatiga $(p=0,001)$. Las puntuaciones de una escala hospitalaria de ansiedad y depresión (Hospital Anxiety and Depression Scale, HADS-D y HADS-A) mejoraron significativamente $(p=0,049)$ entre la primera y la séptima administración de natalizumab. Los pacientes reportaron además menor somnolencia $(p=0,011)$ en la escala de somnolencia de Epworth (Epworth Sleepiness Scale), aunque no se observaron diferencias en la eficiencia del sueño y en el test de latencia múltiple del sueño.

En referencia a los ensayos de medidas de salud percibidas por los pacientes (Tabla 4), el ensayo TRUST [27] evaluó el efecto de natalizumab, demostrando mejorías en la CVRS asociada a incontinencia urinaria determinada con cuestionarios específicos (Urogenital Distress Inventory [UDI-6]; Incontinence Impact Questionnaire [IIQ-7]). A las 24 semanas de tratamiento un $85,7 \%$ de los pacientes reportó mejoras en las puntuaciones del UDI-6 respecto al valor basal $(p<0,001)$ y un $78,6 \%$ en los valores de IIQ-7. Un $64 \%$ de los pacientes experimentó disminución del número semanal de episodios de incontinencia y un $52 \%$ disminución del número de micciones diarias. 
Tabla 3 Características de los estudios de calidad de vida relacionada con la salud en práctica clínica habitual

\begin{tabular}{|c|c|c|c|c|c|}
\hline & Svenningsson 2013 [21] & $\begin{array}{l}\text { Stephenson } 2012 \text { [22], } \\
\text { Kamat } 2009 \text { [19] }\end{array}$ & Putzki 2009 [20] & Zecca [23] & Ratchford [24] \\
\hline $\begin{array}{l}\text { Objetivo principal } \\
\text { del estudio }\end{array}$ & $\begin{array}{l}\text { Estudio TYNERGY: } \\
\text { evaluar la fatiga } \\
\text { relacionada con la EM } \\
\text { durante los } 12 \text { meses } \\
\text { desde el inicio del } \\
\text { tratamiento con } \\
\text { natalizumab }\end{array}$ & $\begin{array}{l}\text { Estudio TOUCH: } \\
\text { evaluar el impacto de } \\
\text { natalizumab en los } \\
\text { PRO dentro de los } 12 \\
\text { meses de tratamiento } \\
\text { con natalizumab }\end{array}$ & $\begin{array}{l}\text { Evaluar la fatiga } \\
\text { relacionada con la } \\
\text { EM después de } 6 \\
\text { meses de } \\
\text { tratamiento con } \\
\text { natalizumab }\end{array}$ & $\begin{array}{l}\text { Evaluar cambio en } \\
\text { la función } \\
\text { cognitiva, fatiga y } \\
\text { CVRS asociada } \\
\text { desescalado de } \\
\text { natalizumab a } \\
\text { IFN } \beta\end{array}$ & $\begin{array}{l}\text { Evaluar la } \\
\text { aparición de } \\
\text { recrudescencias al } \\
\text { final del ciclo de } \\
\text { dosis de } \\
\text { natalizumab }\end{array}$ \\
\hline $\begin{array}{l}\text { Objetivos } \\
\text { secundarios }\end{array}$ & $\begin{array}{l}\text { Evaluar la capacidad de } \\
\text { trabajar, CVRS, } \\
\text { capacidad de dormir, } \\
\text { depresión, velocidad de } \\
\text { caminar, entre otros }\end{array}$ & No evaluados & $\begin{array}{l}\text { Evaluar depresión, } \\
\text { fatiga con otras } \\
\text { metodologías y } \\
\text { bienestar con EVA }\end{array}$ & No evaluados & $\begin{array}{l}\text { Evaluar } \\
\text { diferencias en } \\
\text { fatiga, humor y } \\
\text { CVRS entre el } \\
\text { inicio y el final del } \\
\text { ciclo de dosis de } \\
\text { natalizumab }\end{array}$ \\
\hline Tipo de estudio & $\begin{array}{l}\text { Todos los pacientes } \\
\text { tratados con } \\
\text { natalizumab (única } \\
\text { rama) }\end{array}$ & $\begin{array}{l}\text { Todos los pacientes } \\
\text { tratados con } \\
\text { natalizumab (única } \\
\text { rama) }\end{array}$ & $\begin{array}{l}\text { Todos los } \\
\text { pacientes tratados } \\
\text { con natalizumab } \\
\text { (única rama) }\end{array}$ & $\begin{array}{l}\text { Pacientes con } \\
\text { natalizumab o con } \\
\text { desescalado a } \\
\text { IFN } \beta\end{array}$ & $\begin{array}{l}\text { Estudio transversal } \\
\text { y de cohortes. } \\
\text { Todos los } \\
\text { pacientes tratados } \\
\text { con natalizumab } \\
\text { (única rama) }\end{array}$ \\
\hline Cuestionario & $\begin{array}{l}\text { SF-12 (genérico) y } \\
\text { FSMC (específico de } \\
\text { EM) }\end{array}$ & $\begin{array}{l}\text { SF-12v2 (genérico), } \\
\text { MSIS-29, FS, DS } \\
\text { MOS-Cog, MFIS-5 } \\
\text { (específicos de EM) } \\
\text { mediante entrevista } \\
\text { telefónica }\end{array}$ & $\begin{array}{l}\text { MFIS y FSS } \\
\text { (específicos de } \\
\text { EM) }\end{array}$ & $\begin{array}{l}\text { FSMC, PASAT, } \\
\text { FAMS, EVA-EQ }\end{array}$ & $\begin{array}{l}\text { MSQOL-54, } \\
\text { SF-36, EVA, FSS, } \\
\text { BDI-II }\end{array}$ \\
\hline Periodicidad & Basal y cada tres meses & $\begin{array}{l}\text { Basal y cada tres } \\
\text { meses }\end{array}$ & $\begin{array}{l}\text { Basal y cada tres } \\
\text { meses }\end{array}$ & $\begin{array}{l}\text { Basal, seis meses y } \\
12 \text { meses }\end{array}$ & $\begin{array}{l}\text { Basal y cuatro } \\
\text { semanas (al final } \\
\text { del ciclo de dosis } \\
\text { de natalizumab) }\end{array}$ \\
\hline $\begin{array}{l}\text { Localización del } \\
\text { estudio }\end{array}$ & $\begin{array}{l}27 \text { centros repartidos en } \\
\text { Suecia, Noruega, } \\
\text { Austria y Dinamarca }\end{array}$ & Estados Unidos & Alemania & Suiza & Estados Unidos \\
\hline $\begin{array}{l}\text { Número de } \\
\text { pacientes }\end{array}$ & $\begin{array}{l}164 \text { pacientes, de los } \\
195 \text { iniciales } \\
\text { completaron el } \\
\text { tratamiento de } 12 \text { meses }\end{array}$ & $\begin{array}{l}333 \text { pacientes de } \\
1.275 \text { incluidos en el } \\
\text { análisis completaron } \\
\text { todas las encuestas }\end{array}$ & $\begin{array}{l}\text { El total de } 42 \\
\text { pacientes } \\
\text { incluidos } \\
\text { completaron el } \\
\text { tratamiento de } 6 \\
\text { meses }\end{array}$ & $\begin{array}{l}19 \text { pacientes } \\
\text { incluidos, } 10 \text { en la } \\
\text { rama de } \\
\text { continuación con } \\
\text { natalizumab }\end{array}$ & $\begin{array}{l}82 \text { pacientes } \\
\text { completaron los } \\
\text { cuestionarios de } \\
\text { los } 90 \text { inicialmente } \\
\text { incluidos en el } \\
\text { estudio de cohortes }\end{array}$ \\
\hline $\begin{array}{l}\text { Características } \\
\text { medias de los } \\
\text { pacientes con EM } \\
\text { analizados }\end{array}$ & $\begin{array}{l}71,3 \% \text { mujeres, } 39,7 \\
\text { años y duración media } \\
\text { de EM } 8,8 \text { años EDSS } \\
\text { medio basal: } 3,2 \text { y dos } \\
\text { tercios de los pacientes } \\
\text { sufrió un brote dentro } \\
\text { de los } 6-19 \text { meses } \\
\text { previos a la visita basal }\end{array}$ & $\begin{array}{l}78,1 \% \text { mujeres, } 46,8 \\
\text { años y duración media } \\
\text { de EM 10,6. Grado de } \\
\text { discapacidad evaluado } \\
\text { mediante la escala } \\
\text { DS: DS medio } 3,17\end{array}$ & $\begin{array}{l}60 \% \text { mujeres, } 35,1 \\
\text { años y duración } \\
\text { media de la EM de } \\
7,4 \text { años EDSS } \\
\text { medio } 3,7\end{array}$ & $\begin{array}{l}60 \% \text { mujeres, } 43 \\
\text { años y duración } \\
\text { media de EM } 10 \\
\text { años }\end{array}$ & $\begin{array}{l}70 \% \text { mujeres, } 42,5 \\
\text { años y duración } \\
\text { media de EM 12,7 } \\
\text { años }\end{array}$ \\
\hline $\begin{array}{l}\text { Medida de } \\
\text { resultados }\end{array}$ & $\begin{array}{l}\text { Cambio respecto a la } \\
\text { puntuación basal }\end{array}$ & $\begin{array}{l}\text { Cambio respecto a la } \\
\text { puntuación basal }\end{array}$ & $\begin{array}{l}\text { Cambio respecto a } \\
\text { la puntuación } \\
\text { basal }\end{array}$ & $\begin{array}{l}\text { Cambio respecto a } \\
\text { la puntuación } \\
\text { basal intra grupo, y } \\
\text { comparación entre } \\
\text { las dos ramas de } \\
\text { tratamiento }\end{array}$ & $\begin{array}{l}\text { Cambio respecto a } \\
\text { puntuación basal }\end{array}$ \\
\hline
\end{tabular}


Tabla 3 (Continuación)

\begin{tabular}{|c|c|c|c|c|c|}
\hline & Svenningsson 2013 [21] & $\begin{array}{l}\text { Stephenson } 2012 \text { [22], } \\
\text { Kamat 2009 [19] }\end{array}$ & Putzki 2009 [20] & Zecca [23] & Ratchford [24] \\
\hline Resultados & $\begin{array}{l}\text { La mayor mejoría se } \\
\text { produjo en los } 3 \\
\text { primeros meses de } \\
\text { tratamiento con } \\
\text { natalizumab } \\
\text { Las puntuaciones } \\
\text { motoras y de función } \\
\text { cognitiva mejoraron de } \\
\text { forma significativa } \\
(p<0,0001)\end{array}$ & $\begin{array}{l}\text { Después de } 12 \text { meses } \\
\text { de tratamiento el } \\
69-88 \% \text { de los } \\
\text { pacientes comunicó } \\
\text { mejora o no } \\
\text { empeoramiento en } \\
\text { todas las variables } \\
\text { analizadas. Se } \\
\text { observaron mejoras } \\
\text { significativas a las tres } \\
\text { infusiones en los } \\
\text { cuestionarios de } \\
\text { CVRS tanto general } \\
\text { como específico de la } \\
\text { enfermedad y se } \\
\text { mantuvieron los } 12 \\
\text { meses } \\
\text { La función cognitiva y } \\
\text { la fatiga mejoraron de } \\
\text { forma significativa }\end{array}$ & $\begin{array}{l}\text { La puntuación } \\
\text { referente al } \\
\text { cuestionario MFIS } \\
\text { mejoró de forma } \\
\text { significativa } \\
(p<0,01) \text { en el } \\
\text { sexto mes. Los } \\
\text { pacientes con peor } \\
\text { puntuación basal } \\
\text { en ambos } \\
\text { cuestionarios } \\
\text { fueron los que } \\
\text { tuvieron una } \\
\text { mejoría más } \\
\text { pronunciada }\end{array}$ & $\begin{array}{l}\text { No se observaron } \\
\text { diferencias } \\
\text { significativas en } \\
\text { función cognitiva, } \\
\text { fatiga o CVRS, } \\
\text { aunque sí una } \\
\text { tendencia } \\
\text { coherente a favor } \\
\text { de natalizumab }\end{array}$ & $\begin{array}{l}\text { Se observaron } \\
\text { síntomas de } \\
\text { recrudescencia en } \\
\text { el } 57 \% \text { de los } \\
\text { pacientes }\end{array}$ \\
\hline $\begin{array}{l}\text { Resultados de } \\
\text { variables } \\
\text { secundarias }\end{array}$ & $\begin{array}{l}\text { Todas las variables } \\
\text { secundarias evaluadas } \\
\text { mostraron mejoras } \\
\text { significativas entre el } \\
\text { basal y los } 12 \text { meses de } \\
\text { tratamiento } \\
\text { La variable que no } \\
\text { obtuvo cambios } \\
\text { significativos fue en la } \\
\text { capacidad de caminar, } \\
\text { aunque los datos } \\
\text { obtenidos de esta } \\
\text { variable fueron de baja } \\
\text { calidad }\end{array}$ & No evaluados & $\begin{array}{l}\text { Se observaron } \\
\text { mejorías } \\
\text { significativas en } \\
\text { fatiga (FSS) y en } \\
\text { bienestar (EVA) a } \\
\text { los seis meses } \\
\text { respecto al } \\
\text { momento basal } \\
\text { No hubo variación } \\
\text { en la depresión } \\
\text { respecto al } \\
\text { momento basal a } \\
\text { los tres o seis } \\
\text { meses }\end{array}$ & No evaluados & $\begin{array}{l}\text { Se observaron } \\
\text { reducciones } \\
\text { significativas en } \\
\text { SCF y SCM, } \\
\text { EVA de fatiga } \\
\text { y depresión al final } \\
\text { del ciclo respecto } \\
\text { al momento pico } \\
\text { de natalizumab }\end{array}$ \\
\hline Conclusiones & $\begin{array}{l}\text { Natalizumab, } \\
\text { administrado en la } \\
\text { práctica habitual, puede } \\
\text { mejorar la fatiga } \\
\text { relacionada con la EM } \\
\text { basado en los resultados } \\
\text { de este estudio. } \\
\text { Además, otros } \\
\text { parámetros } \\
\text { relacionados con la } \\
\text { CVRS parecen mejorar } \\
\text { con este tratamiento }\end{array}$ & $\begin{array}{l}\text { Las medidas de PRO } \\
\text { pueden mejorar con el } \\
\text { tratamiento con } \\
\text { natalizumab. Las } \\
\text { mejoras se observaron } \\
\text { en los } 3 \text { primeros } \\
\text { meses y se } \\
\text { mantuvieron en un } \\
\text { periodo de } 12 \text { meses }\end{array}$ & $\begin{array}{l}\text { Además de la } \\
\text { reducción en la } \\
\text { actividad en la } \\
\text { EM, el alivio y } \\
\text { disminución de la } \\
\text { fatiga es un } \\
\text { beneficio relevante } \\
\text { en pacientes con } \\
\text { EM }\end{array}$ & $\begin{array}{l}\text { No se } \\
\text { establecieron } \\
\text { conclusiones } \\
\text { respecto a la rama } \\
\text { que recibió } \\
\text { únicamente } \\
\text { natalizumab }\end{array}$ & $\begin{array}{l}\text { Aproximadamente } \\
\text { 2/3 de los } \\
\text { pacientes tratados } \\
\text { con natalizumab } \\
\text { experimentaron } \\
\text { recrudescencia de } \\
\text { fatiga, debilidad, } \\
\text { discapacidad } \\
\text { cognitiva y motora } \\
\text { al final del ciclo. } \\
\text { Los síntomas } \\
\text { mejoraron tras la } \\
\text { administración de } \\
\text { natalizumab } \\
\text { (mediana de un } \\
\text { día) }\end{array}$ \\
\hline
\end{tabular}

BDI: Beck Depression Inventory; CVRS: calidad de vida relacionada con la salud; DS: Disease Steps; EM: esclerosis múltiple; EVA-EQ: Escala visual analógica de EuroQoL; FAMS: Functional Assessment of Multiple Sclerosis; FS: Functional Status; FSMC: Fatigue Scale for Motor and Cognitive functions; FSS: Fatigue Severity Scale; MFIS: Modified Fatigue Impact Scale; MOS-Cog: Medical Outcomes Scale-Cognitive Functioning; MSIS: Multiple Sclerosis Impact Scale; MSQOL: Multiple Sclerosis Quality of Life; PASAT: Paced Auditory Serial Addition Test; PRO: Patient Reported Outcomes; SF-12: short form 12 items 
Tabla 4 Características de los ensayos con medidas autopercibidas por pacientes

\begin{tabular}{|c|c|c|c|}
\hline & Voloshyna [25] & Sater [26] & Khatri [27] \\
\hline $\begin{array}{l}\text { Objetivo principal del } \\
\text { estudio }\end{array}$ & $\begin{array}{l}\text { Estudio TIMER: evaluar el } \\
\text { impacto de natalizumab en la } \\
\text { deambulación }\end{array}$ & $\begin{array}{l}\text { Estudio NAPS-MS: evaluar la } \\
\text { influencia de } 6 \text { meses de } \\
\text { tratamiento con natalizumab en } \\
\text { medidas de sueño }\end{array}$ & $\begin{array}{l}\text { Estudio TRUST: evaluar el } \\
\text { efecto de } 6 \text { meses de } \\
\text { tratamiento con natalizumab en } \\
\text { la función urinaria }\end{array}$ \\
\hline Objetivos secundarios & No evaluados & $\begin{array}{l}\text { Evaluar cambios en la fatiga, el } \\
\text { humor y la función cognitiva }\end{array}$ & $\begin{array}{l}\text { Evaluar cambios en el número } \\
\text { de episodios semanales de } \\
\text { incontinencia y número diario } \\
\text { de micciones }\end{array}$ \\
\hline Tipo de estudio & $\begin{array}{l}\text { Estudio multicéntrico, } \\
\text { prospectivo, abierto, una rama } \\
\text { (todos los pacientes tratados } \\
\text { con natalizumab) }\end{array}$ & $\begin{array}{l}\text { Estudio prospectivo, abierto, } \\
\text { una rama (todos los pacientes } \\
\text { tratados con natalizumab) }\end{array}$ & $\begin{array}{l}\text { Estudio multicéntrico ( } 2 \text { ), } \\
\text { prospectivo, abierto, una rama } \\
\text { (todos los pacientes tratados } \\
\text { con natalizumab) }\end{array}$ \\
\hline Instrumento & T25FW, T100MW & $\begin{array}{l}\text { PSG, MSLT, ESS, EVA, FSS, } \\
\text { MFIS, HADS-A, HADS-S }\end{array}$ & UDI-6, IIQ-7 \\
\hline Periodicidad & Basal y 24 y 48 semanas & Basal y cada tres meses & Basal y cada cuatro semanas \\
\hline $\begin{array}{l}\text { Localización del } \\
\text { estudio }\end{array}$ & No descrito & Estados Unidos & Estados Unidos \\
\hline Número de pacientes & $\begin{array}{l}224 \text { incluidos, } 215 \text { con al } \\
\text { menos una infusión de } \\
\text { natalizumab }\end{array}$ & 23 pacientes incluidos & $\begin{array}{l}28 \text { pacientes, de } 30 \text { incluidos, } \\
\text { completaron el estudio }\end{array}$ \\
\hline $\begin{array}{l}\text { Características medias } \\
\text { de los pacientes con } \\
\text { EM analizados }\end{array}$ & $\begin{array}{l}\text { 63,7\% mujeres; } 35,1 \text { años y } \\
\text { duración media de EM 9,1 años }\end{array}$ & $\begin{array}{l}\text { 78\% mujeres; } 47,4 \text { años y } \\
\text { duración media de EM 7,5 años }\end{array}$ & $\begin{array}{l}78,6 \% \text { de mujeres; } 49,9 \text { años y } \\
\text { duración media de EM 19,3 } \\
\text { años }\end{array}$ \\
\hline Medida de resultados & Cambio respecto a basal & Cambio respecto a basal & Cambio respecto a basal \\
\hline Resultados & $\begin{array}{l}\text { Se observó incremento } \\
\text { significativo de velocidad a las } \\
24 \text { y } 48 \text { semanas }\end{array}$ & $\begin{array}{l}\text { No se detectaron diferencias } \\
\text { relevantes en la eficiencia del } \\
\text { sueño y test de latencia } \\
\text { múltiple }\end{array}$ & $\begin{array}{l}\text { Se detectaron mejorías } \\
\text { significativas en la función } \\
\text { urinaria desde la semana } \\
\text { número cuatro }\end{array}$ \\
\hline $\begin{array}{l}\text { Resultados de } \\
\text { variables secundarias }\end{array}$ & No evaluadas & $\begin{array}{l}\text { Se observaron mejorías } \\
\text { significativas a los seis meses } \\
\text { en FSS, EVA, ESS y HADS, } \\
\text { pero no en función cognitiva }\end{array}$ & $\begin{array}{l}\text { Se observó disminución } \\
\text { significativa de los episodios de } \\
\text { incontinencia semanal y de las } \\
\text { micciones diarias }\end{array}$ \\
\hline Conclusiones & $\begin{array}{l}\text { Natalizumab incrementa la } \\
\text { velocidad de deambulación en } \\
\text { pacientes con EMRR }\end{array}$ & $\begin{array}{l}\text { Natalizumab no parece mejorar } \\
\text { el patrón de sueño, pero se } \\
\text { asocia con mejorías en fatiga, } \\
\text { somnolencia y humor }\end{array}$ & $\begin{array}{l}\text { Natalizumab se asocia con } \\
\text { mejoría de la CVRS asociada a } \\
\text { incontinencia urinaria }\end{array}$ \\
\hline
\end{tabular}

EM: esclerosis múltiple; ESS: Epworth Sleepiness Scale; EVA: Escala visual analógica; FSS: Fatigue Severity Scale; HADS: Hospital anxiety and Depression Scale; IIQ: Incontinence Impact Questionnaire; MFIS: Modified Fatigue Impact Scale; MSLT: test de latencia múltiple de sueño; PSG: polisomnografía; T25FW: Timed 25-foot walk; T100Mw: timed 100-meter walk; UDI: Urogenital Distress Inventory

En el ensayo TIMER [23] se observaron incrementos significativos $(p<0,0001)$ en la velocidad de desplazamiento en la prueba de $100 \mathrm{~m}$ (timed 100-m walk) a las 24 y 48 semanas del tratamiento con natalizumab comparado con el valor en el estado basal. También se detectaron incrementos estadísticamente significativos ( $p=0,0074)$ en la prueba de desplazamiento de 25 pasos (timed 25-foot walk [T25FW]) a las 24 semanas de tratamiento con natalizumab, pero no en la semana $48(p=0,1643)$.
Se identificó un estudio que evaluaba la recrudescencia de los síntomas de EM durante un ciclo de administración de natalizumab [24], concluyéndose que el CSF del MSQOL 54 era significativamente peor al final del ciclo en comparación con el momento de nivel pico de natalizumab $(62,3$ vs 67,$2 ; p<0,001)$. Resultados similares se observaron en el CSM del MSQOL-54 (69,8 vs 75,5; $p=0,001)$ y también en los valores de la EVA de fatiga (48,9 vs 39,7 ; $p=0,002)$ o en los síntomas depresivos determinados con 
el BDI-II (Beck Depression Inventory-II) (11,6 frente a 9,58; $p=0,006)$.

Los 8 artículos sugieren que natalizumab mejoraba la CVRS relacionada con la EM, así como el resto de variables analizadas, tales como función cognitiva, depresión, movilidad y fatiga.

Tan solo un trabajo que evaluaba el efecto de desescalado de tratamiento, es decir, el cambio a IFN $\beta-1 b$ en pacientes tratados anteriormente con natalizumab [23], no demostró significación estadística en las diferencias de función cognitiva, fatiga y calidad de vida a los 6 y 12 meses respecto al momento basal, en las comparaciones intragrupo del brazo de pacientes que continuaron tratamiento con natalizumab, aunque los autores reportan una tendencia coherente a favor de natalizumab.

\section{Evaluación de la productividad laboral en la práctica clínica habitual}

Se identificó un estudio que evaluaba el efecto de natalizumab sobre la productividad laboral en pacientes con EM en Suecia [28]. El objetivo de este análisis fue estimar el valor monetario del tratamiento con natalizumab en la capacidad de los pacientes con EM para trabajar, basado en la medida directa de las horas semanales trabajadas antes y después de un año de tratamiento con natalizumab. Se incluyeron un total de 202 pacientes con EM, cuya distribución según EDSS era un 38,6\% (78/202) entre 0 y 2; 38,1\% $(77 / 202)$ con un estado EDSS entre 2,5 y 4 y el $23,3 \%$ restante $(47 / 202)$ con EDSS mayor de 4,5. Después de 50 semanas de tratamiento un $72 \%(143 / 202)$ de los pacientes mantuvo estable su capacidad de trabajar, mientras que un $22 \%(46 / 202)$ de los pacientes experimentó aumento y un $6 \%(13 / 202)$ de los pacientes disminución de su capacidad de trabajar. De forma global, los pacientes aumentaron de forma significativa la capacidad de trabajar con un aumento medio de 3,3 horas semanales por paciente, correspondiendo a una productividad laboral anual de $3.216 €$ (referentes a euros del año 2007). Cuando el análisis se restringió a pacientes que tenían un empleo (72\%) y pensión por discapacidad o beneficios sociales, esta ganancia en productividad aumentó hasta $8.185 €$ anuales por paciente. Asimismo, se asoció un beneficio significativamente mayor en los pacientes tratados con natalizumab con menor duración de la enfermedad ( $p=0,025)$, o en aquellos que tuvieran una edad comprendida entre los 25 y 35 años $(p=0,002)$. Por esta razón, los autores sugerían que sería más beneficioso empezar de manera precoz con terapias eficientes en pacientes con EM.

\section{Análisis combinados o post-hoc de estudios fase III de eficacia de natalizumab}

Se realizó un análisis sobre un subgrupo de pacientes del estudio pivotal AFFIRM con un estado basal de la enferme- dad con EDSS $\geq 2,0$. De los 620 pacientes analizados 203 se trataron con placebo y 417 con natalizumab [30].

Se evaluó la calidad de vida en estos pacientes mediante el CSF y CSM del cuestionario SF-36. El tratamiento con natalizumab mejoró las puntuaciones tanto del CSF ( $p=0,003)$ como del CSM $(p=0,011)$ en comparación con placebo durante los dos años del estudio. Los pacientes que recibieron tratamiento con natalizumab tuvieron significativamente mayores cambios desde las puntuaciones basales en 6 de los 8 dominios individuales del SF-36 comparadas con placebo $(p<0,05)$.

Adicionalmente se agruparon en una única evaluación todos los pacientes $(n=2.113)$ incluidos en los estudios AFFIRM (natalizumab administrado en monoterapia) y SENTINEL (administrado en combinación con IFN $\beta$-1a IM) [29]. En ambos estudios se administró el SF-36 (englobando tanto el CSF como el CSM) y una EVA para la medida de la CVRS, en la visita basal y en las semanas 24, 52 y 104. Como sucede en todos los pacientes con EM, la puntuación basal fue significativamente menor que la obtenida en la población general, que también va correlacionada con la puntuación EDSS del paciente. La administración de natalizumab mejoró significativamente las puntuaciones del SF-36, tanto el CSF como el CSM, así como la escala de EVA. Los pacientes tratados con natalizumab tuvieron mejoras significativas del CSF del SF-36 desde la semana 24 y en todos los cortes temporales $(p<0,05)$. Un mayor porcentaje de pacientes del estudio AFFIRM tratados con natalizumab mejoró tanto en el dominio físico en comparación con placebo (24,9\% frente a $16,8 \%)$, como en el mental (28,5\% frente a 21,6\%). Asimismo, un menor número empeoró comparado con placebo en los dominios tanto físico $(18,0 \%$ frente a $25,15 \%$ ) como mental (17,9\% frente a $22,2 \%)$.

Un análisis post-hoc del estudio AFFIRM [31] evaluó la relación entre capacidad y agudeza visual, de bajo y alto contraste, determinadas mediante una subescala del cuestionario de calidad de vida en EM Impact of visual Impairment Scale (IVIS) y optotipos con tipografías de Sloan, con la CVRS medida con el SF-36 y una EVA. A los dos años el tratamiento con natalizumab se asoció con una reducción relativa frente a placebo de la progresión de la agudeza visual de bajo y alto contraste, del $32 \%$ y $34 \%$, respectivamente. Se observó una correlación lineal entre bajas puntuaciones de IVIS y del CSF del SF-36, con valores reducidos de función visual a las 52 y a las 104 semanas de tratamiento.

Los análisis post-hoc del estudio AFFIRM [23] y AFFIRM junto a SENTINEL [32], que evaluaron la deambulación en los pacientes tratados con natalizumab, detectaron un incremento de la proporción de pacientes con mejoras de la velocidad en el test T25FW del 78\% (12,3\% frente a $6,9 \% ; p=0,00133$ ) en comparación con placebo tras dos años de tratamiento [23], observando una correlación significativa entre este incremento y mejorías de la CVRS a 
nivel del CSF del SF-36 $(p<0,005)$ en todos los grupos de tratamiento.

\section{Discusión}

Tradicionalmente siempre se ha evaluado la eficacia del tratamiento en EM mediante técnicas de imagen, por resonancia magnética y medidas clínicas como la tasa anual de brotes y el nivel de discapacidad, determinado con la puntuación de la EDSS. Sin embargo, es también necesaria una aproximación más centrada en el paciente, teniendo en cuenta, por una parte, que los síntomas de la enfermedad podrán afectar a las actividades básicas de su día a día. Por otra parte, los tratamientos actualmente disponibles son paliativos, buscando frenar el curso natural de la enfermedad, sin que exista, hoy por hoy, cura para la EM y, por lo tanto, son tratamientos que se van a administrar de forma crónica. La CVRS es un factor fundamental a tener en cuenta cuando se evalúan los efectos de cualquier tratamiento en un paciente con EM, ya que constituye un importante indicativo de salud.

La presente revisión confirma que natalizumab aporta mejoras en la CVRS medida tanto en AVAC como respecto a los niveles basales de los pacientes antes de recibir dicho tratamiento, evaluado mediante cuestionarios de CVRS específicos. Así mismo, se evaluaron distintos factores tales como función cognitiva y depresión, siendo la fatiga el más evaluado y que mejoró de forma significativa en todos los estudios analizados, así como la deambulación, la incontinencia urinaria y el sueño.

En las 4 modelizaciones identificadas se tuvo en cuenta el riesgo de sufrir LMP, concluyéndose que natalizumab es un fármaco muy eficaz en el tratamiento de la EM, que aporta más beneficios en salud y CVRS que las opciones terapéuticas frente a las cuales se comparó, aun teniendo en cuenta el riesgo real de sufrir LMP. A pesar de no ser estudios en la vida real, estos análisis nos ayudan a simular lo que ocurriría en un paciente con EM que recibe tratamiento con natalizumab en distintos horizontes temporales y comparar estos resultados con otras alternativas terapéuticas de interés.

Así mismo se detectó un estudio que evaluaba el valor monetario en términos de productividad laboral de pacientes con EM que recibieron tratamiento con natalizumab durante un año, concluyendo que iniciar de forma precoz el tratamiento con este fármaco tenía una repercusión económica considerable [28]. Cabe destacar la importancia de este análisis, ya que como se ha mencionado anteriormente la EM es una enfermedad que afecta mayoritariamente a pacientes jóvenes y en edad laboralmente activa y que, debido a esta enfermedad, por la presencia de brotes o progresión de la discapacidad genera importante influencia en el funcionamiento personal, laboral y social del paciente. Según el estudio que analizó este aspecto concreto, un inicio temprano de terapias eficientes tendría una repercusión financiera importante, ya que aumentaría de forma significativa la capacidad laboral de estos pacientes con el consiguiente impacto económico y social.

Los instrumentos para evaluar los cambios en la calidad de vida observados en esta revisión fueron muy diversos, tanto cuestionarios validados y estandarizados genéricos como otros específicos de la EM. La importancia de evaluar este parámetro con métodos estandarizados radica en la importancia de objetivar de forma reproducible la mejoría o empeoramiento de la CVRS. Por este motivo, estudios adicionales detectados en esta revisión que analizaban la CVRS sin evaluarla mediante métodos estandarizados fueron excluidos. Concretamente, en una de ellas la CVRS se evaluó mediante entrevista telefónica a 20 pacientes que al menos hubieran recibido 6 infusiones de natalizumab [37]. Los resultados del estudio se facilitaban a nivel cualitativo con impresiones y comentarios de los pacientes, y las conclusiones fueron que la mayoría de los pacientes experimentó mejoría en al menos un síntoma referente a la calidad de vida. El estudio TORONTO [38], observacional retrospectivo, cuyo objetivo era evaluar la eficacia de natalizumab sobre 146 pacientes, analizaba también los efectos subjetivos de natalizumab preguntando a los pacientes cómo había cambiado su vida desde el inicio del tratamiento con dicho medicamento, y se obtuvo que un $85 \%$ de dichos pacientes notó mejoría, impactando en aumento de la energía, función cognitiva/memoria, fuerza y equilibrio, entre otros.

Como posibles limitaciones del estudio, aparte de las propias de cada uno de los trabajos revisados, cabe destacar que, como ocurre en todas las revisiones sistemáticas, existe la posibilidad de no incluir todos los estudios relacionados con el objetivo del mismo por no estar etiquetados correctamente en la base de datos donde se realizó la búsqueda, y por tanto no poder ser identificados. Asimismo, los términos de búsqueda tanto de calidad de vida como de productividad laboral no están etiquetados como términos MeSH, por lo que es posible que pudieran no haber sido identificados. Para minimizar el sesgo de ambos hechos se realizó una revisión manual de las referencias bibliográficas de los trabajos incluidos, así como de revisiones sistemáticas identificadas.

Analizar los cambios en la CVRS y productividad laboral en los pacientes con EM tras recibir tratamiento con natalizumab ha sido el fin principal de esta revisión. A pesar de la diversidad de metodologías entre los estudios incluidos, los resultados ponen de manifiesto que natalizumab es una opción de tratamiento que mejora la calidad de vida y la productividad laboral de los pacientes.

Conflicto de intereses V. Casado Ruiz y R. Ginestal han recibido honorarios de Biogen por su asesoría en el desarrollo de este proyecto. 
M. Echave, I. Oyagüez y M.A. Casado son empleados de Pharmacoeconomics \& Outcomes Research Iberia (PORIB), una consultora especializada en evaluación económica de intervenciones sanitarias que ha recibido financiación de Biogen para el desarrollo del presente análisis y la redacción del presente manuscrito.

Open Access This article is distributed under the terms of the Creative Commons Attribution-NonCommercial 4.0 International License (http://creativecommons.org/licenses/by-nc/4.0/), which permits any noncommercial use, distribution, and reproduction in any medium, provided you give appropriate credit to the original author(s) and the source, provide a link to the Creative Commons license, and indicate if changes were made.

\section{Bibliografía}

1. Pugliatti M, Rosati G, Carton H, et al. The epidemiology of multiple sclerosis in Europe. Eur J Neurol. 2006;13(7):700-22.

2. Agencia Canadiense de Evaluación de Medicamentos y Tecnologías Sanitarias (Canadian Agency for Drugs and Technologies in Health) CADTH [consultado 25 Jun 2014]. Disponible en: http://www.cadth.ca/en/products/therapeutic-reviews/relapsingremit-multiple-sclerosis/reports

3. Meyer-Moock S, Feng YS, Maeurer M, et al. Systematic literature review and validity evaluation of the Expanded Disability Status Scale (EDSS) and the Multiple Sclerosis Functional Composite (MSFC) in patients with multiple sclerosis. BMC Neurol. 2014; $14: 58$

4. Organización Mundial de la Salud. Atlas Multiple Sclerosis Resources in the World 2008 [consultado Jun 2014]. Disponible en: http://www.who.int/mental_health/neurology/Atlas_MS_WEB. pdf

5. McDowell TY, Amr S, Langenberg P, et al. Time of birth, residential solar radiation and age at onset of multiple sclerosis. Neuroepidemiology. 2010;34(4):238-44.

6. Busche KD, Fisk JD, Murray TJ, Metz LM. Short term predictors of unemployment in multiple sclerosis patients. Can J Neurol Sci. 2003;30(2):137-42.

7. Fattore G, Lang M, Pugliatti M. The Treatment experience, burden, and unmet needs (TRIBUNE) study-measuring the socioeconomic consequences of Multiple Sclerosis. Mult Scler. 2012;18(2 Suppl):5-6

8. Trisolini M. Global economic impact of multiple sclerosis. 2010 [consultado 25 Jun 2014]. Disponible en: http://www.msif.org/includes/documents/cm_docs/2011/g/global_ economic_impact_of_ms.pdf?f=1

9. Guyatt GH, Feeny DH, Patrick DL. Measuring health-related quality of life. Ann Intern Med. 1993;118(8):622-9.

10. Fernández-López JA, Hernández-Mejía R, Siegrist J. El perfil de calidad de vida para enfermos crónicos (PECVEC): un método para evaluar bienestar y funcionalismo en la práctica clínica. Aten Prim. 2001;28(10):680-9.

11. Ficha técnica de natalizumab [consultado 25 Jun 2014]. Disponible en: http://www.ema.europa.eu/docs/es_ES/document_library/ EPAR_-_Product_Information/human/000603/WC500044686.pdf

12. Polman $\mathrm{CH}$, O'Connor PW, Havrdova E, et al. A randomized, placebo-controlled trial of natalizumab for relapsing multiple sclerosis. N Engl J Med. 2006;354(9):899-910.

13. Rudick RA, Stuart WH, Calabresi PA, et al.; SENTINEL Investigators Natalizumab plus interferon beta-1a for relapsing multiple sclerosis. N Engl J Med. 2006;354(9):911-23.

14. Nota informativa de la AEMPS sobre natalizumab (Tysabri ${ }^{\circledR}$ ) y leucoencefalopatía multifocal progresiva: actualización de la información [consultado 25 Jun 2014]. Disponible en: http://www.
aemps.gob.es/informa/notasInformativas/medicamentosUso Humano/seguridad/2010/NI_2010-02_natalizumab_tysabri.htm

15. Dorsey ER, Thompson JP, Noyes K, et al. Quantifying the risks and benefits of natalizumab in relapsing multiple sclerosis. Neurology. 2007;68(18):1524-8.

16. Thompson JP, Noyes K, Dorsey ER, et al. Quantitative risk-benefit analysis of natalizumab. Neurology. 2008;71(5):357-64.

17. Walker A, Watson C, Alexopoulos ST, et al. A benefit-risk analysis of natalizumab in the treatment of patients with multiple sclerosis when considering the risk of progressive multifocal leukoencephalopathy. Curr Med Res Opin. 2014;30(4):629-35.

18. Campbell JD, McQueen RB, Miravalle A, et al. Comparative effectiveness of early natalizumab treatment in JC virusnegative relapsing-remitting multiple sclerosis. Am J Manag Care. 2013;19(4):278-85.

19. Kamat SA, Rajagopalan K, Stephenson JJ, Agarwal S. Impact of natalizumab on patient-reported outcomes in a clinical practice setting: a cross-sectional survey. Patient. 2009;2(2):105-12.

20. Putzki N, Yaldizli O, Tettenborn B, Diener HC. Multiple sclerosis associated fatigue during natalizumab treatment. J Neurol Sci. 2009;285(1-2):109-13.

21. Svenningsson A, Falk E, Celius EG, et al.; Tynergy Trial Investigators. Natalizumab treatment reduces fatigue in multiple sclerosis. Results from the TYNERGY trial; a study in the real life setting. PLoS One. 2013;8(3):e58643.

22. Stephenson JJ, Kern DM, Agarwal SS, et al. Impact of natalizumab on patient-reported outcomes in multiple sclerosis: a longitudinal study. Health Qual Life Outcomes. 2012;10:155.

23. Zecca C, Riccitelli GC, Calabrese P, et al. Treatment satisfaction, adherence and behavioral assessment in patients de-escalating from natalizumab to interferon $\beta$. BMC Neurol. 2014;14:38.

24. Ratchford JN, Brock-Simmons R, Augsburger A, et al. Multiple sclerosis symptom recrudescence at the end of the natalizumab dosing cycle. Int J MS Care. 2014;16(2):92-8.

25. Voloshyna N, Havrdová E, Hutchinson M, et al. Natalizumab improves ambulation in relapsing-remitting multiple sclerosis: results from the prospective TIMER study and a retrospective analysis of AFFIRM. Eur J Neurol. 2015;22(3):570-7.

26. Sater RA, Gudesblatt M, Kresa-Reahl K, et al. NAPS-MS: natalizumab effects on parameters of sleep in patients with multiple sclerosis. Int J MS Care. 2016;18(4):177-82.

27. Khatri BO, Foley JF, Fink J, et al. The TRUST (evaluation of bladder function in relapsing-remitting multiple sclerosis patients treated with natalizumab) observational study. Int J MS Care. 2014;16(1):40-7.

28. Olofsson S, Wickström A, Häger Glenngård $\mathrm{A}$, et al. Effect of treatment with natalizumab on ability to work in people with multiple sclerosis: productivity gain based on direct measurement of work capacity before and after 1 year of treatment. BioDrugs. 2011;25(5):299-306.

29. Rudick RA, Miller D, Hass S, et al, AFFIRM and SENTINEL Investigators. Health-related quality of life in multiple sclerosis: effects of natalizumab. Ann Neurol. 2007;62(4):335-46.

30. Phillips JT, Giovannoni G, Lublin FD, et al. Sustained improvement in expanded disability status scale as a new efficacy measure of neurological change in multiple sclerosis: treatment effects with natalizumab in patients with relapsing multiple sclerosis. Mult Scler. 2011;17(8):970-9.

31. Chahin S, Balcer LJ, Miller DM, et al. Vision in a phase 3 trial of natalizumab for multiple sclerosis: relation to disability and quality of life. J Neuroophthalmol. 2015;35(1):6-11.

32. Cohen JA, Krishnan AV, Goodman AD, et al. The clinical meaning of walking speed as measured by the timed 25 -foot walk in patients with multiple sclerosis. JAMA Neurol. 2014;71(11):138693.

33. Rubio-Terrés C. Introducción a los modelos de Markov en el análisis farmacoeconómico. Farm Hosp. 2000;24:241-7. 
34. Soto Alvarez J. Evaluación económica de medicamentos y tecnologías sanitarias. Madrid: Springer SBM Spain, SAU; 2012.

35. Bloomgren G, Richman S, Hotermans C, et al. Risk of natalizumab-associated progressive multifocal leukoencephalopathy. N Engl J Med. 2012;366:1870-80.

36. Yousry TA, Major EO, Ryschkewitsch C, et al. Evaluation of patients treated with natalizumab for progressive multifocal leukoencephalopathy. N Engl J Med. 2006;354:924-33.
37. Miller CE, Karpinski M, Jezewski MA. Relapsing-remitting multiple sclerosis patients' experience with natalizumab: a phenomenological investigation. Int J MS Care. 2012;14(1):39-44.

38. Krysko KM, O'Connor PW. The TORONTO observational study of natalizumab in multiple sclerosis. Can J Neurol Sci. 2011;38(3):422-8. 